\title{
From filopodia to synapses: the role of actin-capping and anti-capping proteins
}

\author{
Elisabetta Menna, ${ }^{1}$ Giuliana Fossati, ${ }^{1}$ Giorgio Scita ${ }^{2}$ and Michela Matteoli ${ }^{1,3}$ \\ ${ }^{1}$ Department of Medical Pharmacology and CNR Institute of Neuroscience, University of Milan, Milano, Italy \\ ${ }^{2}$ IFOM, FIRC Institute of Molecular Oncology Foundation at IFOM-IEO Campus, Milano, Italy \\ ${ }^{3}$ IRCCS Don Gnocchi, Milano, Italy
}

Keywords: BDNF, capping proteins, filopodia formation, neuritogenesis, synaptogenesis

\begin{abstract}
Actin-capping and anti-capping proteins are crucial regulators of actin dynamics. Recent studies have indicated that these proteins may be heavily involved in all stages of synaptogenesis, from the emergence of filopodia, through neuritogenesis and synaptic contact stabilization, to the structural changes occurring at the synapse during potentiation phenomena. In this review, we focus on recent evidence pointing to an active role of actin-capping and anti-capping proteins in orchestrating the processes controlling neuronal connectivity and plasticity.
\end{abstract}

\section{Introduction}

Neural circuits are defined by the structure of axons and dendrites, with single axons contacting and controlling the function of multiple targets, and individual dendrites integrating inputs from several sources. The molecular processes involved in establishing proper neuronal connectivity are not exclusively activated during brain development, because, even in the adult brain, continuous remodelling, accompanied by synapse formation and elimination, underlies the process of memory formation (Chklovskii et al., 2004; Bruel-Jungerman et al., 2007; Fu \& Zuo, 2011). Deciphering the molecular mechanisms by which neurite extension and synaptogenesis occur is therefore critical to our understanding of brain ontogenesis, synaptic remodelling, and plasticity.

It is widely accepted that filopodia, which are highly motile, narrow extensions containing bundles of filamentous actin, play important roles at initial stages of synaptogenesis (Fiala et al., 1998; Craig et al., 2006; Geraldo \& Gordon-Weeks, 2009). Also, growth and stabilization of filopodia for the formation of new synaptic contacts occur during long-term potentiation (Luscher et al., 2000; Jourdain et al., 2003; Nikonenko et al., 2003). Filopodia emerge from all neuronal compartments: those extending at the tips of axonal growth cones mainly mediate neurite navigation and axonal pathfinding (Koleske, 2003). Filopodia emerging from developing axons, which are characterized by the presence of actively recycling synaptic vesicles, are thought, instead, to represent precursors of presynaptic boutons (Chang \& De Camilli, 2001; Matteoli et al., 2004). On the other hand, dendritic filopodia may serve as precursors of new spines in the context of activity-dependent synaptogenesis (Jontes \& Smith, 2000; Wong et al., 2000; Portera-Cailliau et al., 2003; Yuste \& Bonhoeffer, 2004; Knott \& Holtmaat, 2008). The transition from filopodia to spines upon contact with a presynaptic bouton has been directly

Correspondence: M. Matteoli, as above.

E-mail: michela.matteoli@unimi.it

Received 15 July 2011, revised 9 September 2011, accepted 11 September 2011 demonstrated (Ziv \& Smith, 1996; Kayser et al., 2008; Heiman \& Shaham, 2010; Arstikaitis et al., 2011).

It is generally thought that filopodial actin filaments originate from the lamellipodium, a flat membrane protrusion that is almost invariably seen at the leading edge of migratory cells. The diverse morphological features of lamellipodia and filopodia are mirrored by their architecturally diverse actin backbones. An extended meshwork of actin filaments of variable length and orientation supports the former protrusions, whereas filopodia are composed of bundles of long and linear actin filaments.

A number of actin-binding proteins have been implicated in regulating the equilibrium between filopodia and lamellipodia (Gupton et al., 2007; Drees \& Gertler, 2008; Le Clainche \& Carlier, 2008). In particular, the formation of lamellipodia mainly involves the activity of a minimal key set of actin regulatory proteins (see below), and of the Arp2/3 complex, a branched actin filament nucleator that works in concert with actin-capping proteins. These latter proteins, by binding to the barbed ends of densely packed, plasma membrane-localized actin filaments, control not only their lifetime, but also the architecture of the resulting meshwork (Akin \& Mullins, 2008). Filopodial initiation and extension requires, instead, the coordinated and balanced activities of actin-capping and anti-capping proteins that, together with linear filament elongators, including formin and vasodilator-stimulated phosphoprotein (VASP) family proteins, promote the formation of long filaments; the latter then converge to form bundles, which subsequently become tightly cross-linked through the action of proteins such as fascin (Mejillano et al., 2004; Mogilner \& Rubinstein, 2005; Drees \& Gertler, 2008; Mattila \& Lappalainen, 2008; Ridley, 2011) (Fig. 1).

Given that the mechanisms regulating the formation of neuronal filopodia directly impact on neuronal connectivity and network plasticity, we focus here on recent evidence pointing to an active role of actin-capping and anti-capping proteins in orchestrating the formation of neuronal filopodia, as well as in controlling neuritogenesis and spine morphology. 


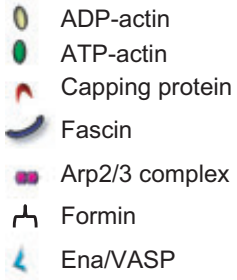

$<$ EnaNASP

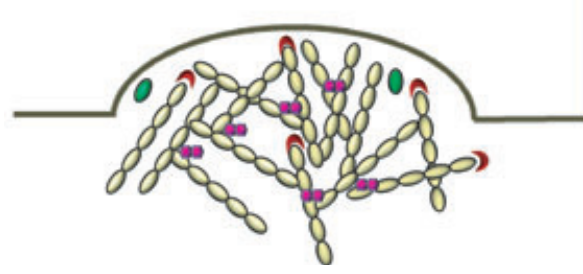

FIG. 1. Actin-binding proteins regulate the equilibrium between lamellipodia and filopodia. Left: the lamellipodial structure is maintained by the concerted actions of actin-capping and branching proteins. Right: filopodia formation is favoured by the removal of capping proteins and by the concomitant activity of actin-cross-linking (bundling) proteins.

\section{The formation of filopodia in neurons: a role for actin-capping and anti-capping proteins}

The core structural and dynamic components of filopodia are actin filaments, whose dynamic formation and topological organization are controlled by ensembles of actin-binding proteins. The actin regulatory machinery responsible for filopodia consists of different classes of proteins, such as F-actin nucleators (e.g. Arp2/3, formins), filament bundlers (e.g. fascin), membrane-deforming factors (e.g. BAR domain proteins), regulators of actin polymerization (e.g. Ena/VASP family proteins, profilin, and actin-capping proteins) or disassembly (e.g. $\mathrm{ADF} /$ cofilin), and actin-associated motors (e.g. myosin II and myosin X) (Ono, 2007; Le Clainche \& Carlier, 2008; Chesarone \& Goode, 2009).

The mechanism of filopodia formation is a controversial topic (Svitkina et al., 2003; Vignjevic et al., 2006; Gupton et al., 2007; Mattila \& Lappalainen, 2008; Faix et al., 2009; Mellor, 2010). Two alternative models have been suggested: the convergent elongation model proposes that Arp2/3-seeded actin filaments are elongated by factors, such as Ena/VASP family proteins, that also protect them from capping, and are assembled into filopodial bundles by fascin; in contrast, the de novo nucleation model proposes that linear filament nucleators and elongators, such as formin and Ena/VASP family proteins, assemble into submembranous complexes, thereby promoting the processive elongation of parallel actin filaments, which are cross-linked into filopodial actin bundles. It must be pointed out that these two models are not necessarily exclusive, as both mechanisms may operate in cells in response to distinct sets of conditions, such as the abundance of specific cytoskeletal components, different signalling pathways, and diverse microenvironmental conditions, including the composition of the extracellular matrix in which cells are embedded.

The activity of actin-capping proteins, according to the convergent elongation model, is crucial for controlling the resultant architectural organization of actin in these protrusions. When the capping activity is high, newly nucleated branched filaments become rapidly capped; this also causes a local increase in the concentrations of available actin monomers, which further feed Arp $2 / 3$ nucleation/branching activity, ultimately promoting the generation of a dense and highly branched dendritic array of short actin filaments at the leading edge of lamellipodia. Conversely, when capping activity is low, local monomer availability is reduced, as G-actin becomes incorporated into long and uncapped actin filaments (Mogilner \& Rubinstein, 2005; Akin \& Mullins, 2008; Korobova \& Svitkina, 2008). Factors such as VASP family members or ill-defined components of the filopodia tip complex may then promote the transient association of actin filaments, which can be further stabilized by other bundlers, such as fascin, thus permitting the formation of actin bundles and filopodia (Mogilner \& Rubinstein, 2005). According to the de novo nucleation model, formins (Pellegrin \& Mellor, 2005; Schirenbeck et al., 2005; Yang et al., 2007; Block et al., 2008) or VASP tetramers, particularly when clustered along the plasma membrane (Breitsprecher et al., 2008, 2011; Hansen \& Mullins, 2010), may be responsible for promoting filopodial initiation. Also in this case, however, filaments must be protected from cappers, which have been shown, in the case of the capping protein $(\mathrm{CP})$, to compete, either directly or indirectly, with VASP as well as with formins for barbed-end binding (Breitsprecher et al., 2008).

Actin-capping proteins appear to play a role in regulating lamellipodia and filopodia formation in neurons. Gelsolin, for example, which severs actin filaments in a calcium-dependent manner and caps the plus ends of severed filaments, preventing the addition of actin monomers, appears to function in the initiation of filopodial retraction and in its smooth progression ( $\mathrm{Lu}$ et al., 1997). Another example is the actin-capping and regulatory protein Eps8, which is regulated, unlike gelsolin, not by calcium levels, but by protein-protein interactions and phosphorylation (see below). Eps8 is the prototype of a family of proteins involved in the regulation of actin remodelling (Offenhauser et al., 2004). It is able to activate Rac, which in turn regulates the actin cytoskeleton. Moreover, Eps8 directly controls actin dynamics and the architecture of actin structures by capping barbed ends and cross-linking actin filaments, respectively (Croce et al., 2004; Disanza et al., 2006). The barbed-end-capping activity of Eps8 resides in its conserved C-terminal effector domain, and it is functional when the protein is associated with Abil (Disanza et al., 2004). Conversely, Eps8 must associate with insulin receptor tyrosine kinase substrate of $53 \mathrm{kDa}$ (IRSp53), also known as binding partner of the brain-specific angiogenesis inhibitor 1 (Abbott et al., 1999; Oda et al., 1999), to efficiently cross-link actin filaments (Disanza et al., 2006). These multiple actin regulatory roles of Eps8 in vitro are reflected by the observation that, in vivo, Eps8 is required for optimal actin-based motility, intestinal morphogenesis, and filopodia-like extension (Croce et al., 2004; Disanza et al., 2004 and 2006). In hippocampal neurons, overexpression of Eps8 causes the formation of flat, actin-rich protrusions along axons, which resemble lamellipodia extensions; on the other hand, protein silencing leads to increased filopodia formation in the axonal and dendritic compartments (Menna et al., 2009; Fig. 2A). Using fluorescence recovery after photobleaching measurements of actin in developing neurons, which allows measurement of fluorescence recovery as an indication of the movement of fluorophores after perturbation by photobleaching, we show here that the process of Eps8 removal is accompanied by an alteration in actin dynamics at the growth cone, as indicated by the reduction in the half-time of recovery $\left(t_{1 / 2}\right)$ after photobleaching (Fig. 2B and C). Interestingly, the increased axonal filopodia formation in Eps8 null hippocampal neurons is significantly reduced upon interference with VASP functions through dominant-negative approaches, suggesting functional competition between the capping activity of Eps8 and the actin-regulatory properties of VASP (Menna et al., 2009). 


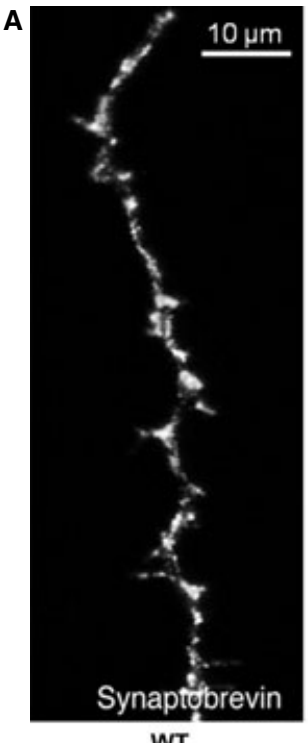

WT

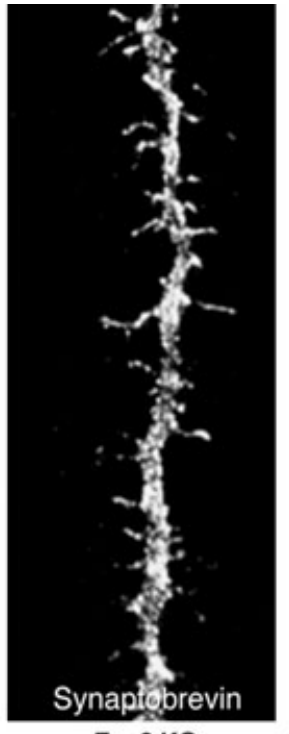

Eps8 KO
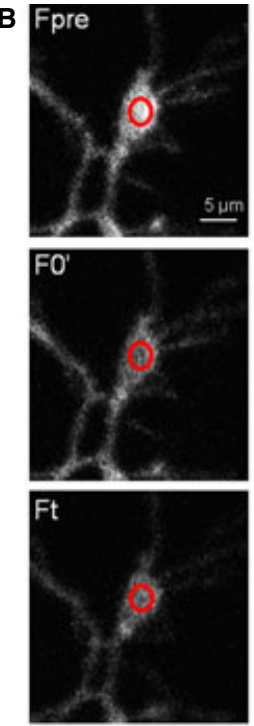
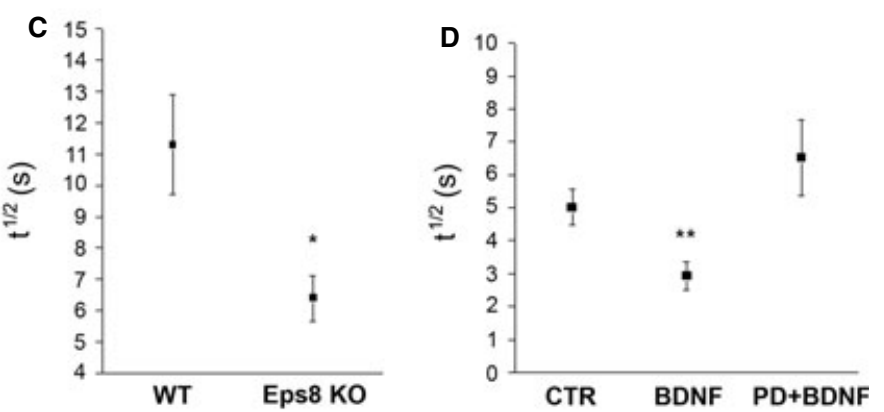

FIG. 2. Removal of capping proteins favours filopodia formation in neurons: the case of Eps8. (A) Increased number of filopodia in neurons from Eps8 knockout (KO) mice relative to wild-type (WT) mice. Staining for the synaptic vesicle synaptobrevin is shown. Reprinted from Menna et al. (2009). (B) An axonal growth cone of 6 days in vitro hippocampal neurons expressing yellow fluorescent protein-actin selectively photobleached by high-intensity laser light (514 nm). Fluorescence recovery was followed by collecting images at rates of one image every $500 \mathrm{~ms}$ for $2.5 \mathrm{~min}$. Scale bar: $5 \mu \mathrm{m}$. The red circle represents the region of interest used for both the photobleaching and the fluorescence recovery analysis. For interpretation of color references in figure legend, please refer to the Web version of this article. (C) Actin dynamics at the axonal growth cone are significantly accelerated in the absence of the actin-capping protein Eps8. Number of growth cones: WT, 31; and and Eps8 KO, 25. (Mann-Whitney rank sum test, $P=0.017$.) (D) Actin dynamics, measured at the axonal growth cone, are significantly accelerated after BDNF treatment, which increases filopodia formation (see text for details). The increase in actin dynamics is prevented by treatment with the MAPK blocker PD98059 (PD). Number of growth cones: control (CTR), 18; BDNF, 18; PD + BDNF, 16. (Kruskal-Wallis one way ANOVA on ranks, $P=0.002)$. $* P<0.05 ; * * P<0.01$.

\section{The regulation of capping activity in filopodia formation: intrinsic and extrinsic cues}

In a simplified view, actin-capping proteins can be seen as inhibitors, whereas bundling proteins are among the necessary components for filopodia formation. Consistent with this picture, removal of $\mathrm{CP}$, even in non-neuronal cells, causes an increase in the number of filopodia (Mejillano et al., 2004; Applewhite et al., 2007), whereas cells devoid of the actin cross-linker fascin show a reduced amount of filopodia (Vignjevic et al., 2006). Eps8 can efficiently cap barbed ends when bound to Abi-1 (Disanza et al., 2004), whereas it cross-links actin filaments, particularly when it associates with IRSp53, a potent inducer of filopodia via its ability to bind actin filaments and deform the plasma membrane (Scita et al., 2008). Consistent with its dual function, the role of Eps8 in filopodia formation is cell contextdependent. In HeLa and other epithelial cell lines, overexpression of Eps8 promotes the formation of filopodia (Disanza et al., 2006), whereas in primary hippocampal neurons, genetic removal of Eps8 increases the formation of axonal and dendritic filopodia (Menna et al., 2009). By the use of a combination of in vivo and in vitro experiments, together with a system of ordinary differential equations, a mathematical model was recently generated that allowed us to explain how filopodia are formed in different cellular contexts (Vaggi et al., 2011). This study showed that the biochemical activities of Eps8 as a capper and bundler are a function of the dynamic interactions established by Eps8, IRSp53 and VASP with actin filaments. Eps8 therefore represents a molecular switch in the transduction of signalling, directing the cells towards either reduced or increased formation of filopodia, mainly depending on the relative concentrations of the components of the protein network underlying filopodia formation (Vaggi et al., 2011).
One open question is how extracellular cues are translated into changes in the protein network, which operate in a dynamic interplay to control the actin cytoskeleton remodelling that is important for filopodia formation. Different cues in the extracellular environment have been found to modulate the dynamics of lamellipodia and filopodia, inducing either increased protrusions or collapse (McAllister, 2007; Lundquist, 2009; Mai et al., 2009; Valiente \& Martini, 2009). However, the signalling pathways by which these stimuli impact on actin dynamics are largely unknown. In sensory neurons, nerve growth factor (NGF) promotes the formation of axonal filopodia and branches. With the use of chicken sensory neurons and live imaging of enhanced yellow fluorescent protein-actin dynamics, it has been found that NGF induces the formation of microdomains of phosphatidylinositol 3,4,5-trisphosphate and actin patches from which filopodia could emerge. However, NGF does not directly promote the emergence of filopodia from patches themselves (Ketschek \& Gallo, 2010). The cytoskeletal proteins downstream of NGF-phosphoinositide 3-kinase signalling are presently not characterized, although the bundling protein fascin might be implicated (Ketschek et al., 2011).

More detailed insights into the signal transduction process by which an extracellular cue may regulate the formation of axonal filopodia have been obtained for the neurotrophin brain-derived neurotrophic factor (BDNF), a protein secreted by synaptic targets to modulate neuronal survival and differentiation, and which induces filopodia formation (Fass et al., 2004; Gehler et al., 2004; Chen et al., 2006). In particular, a direct link between extracellular BDNF and axonal filopodia formation was shown to involve Eps8 capping activity. BDNF, through the activation of Trk receptor tyrosine kinases and activation of mitogen-activated protein kinase (MAPK) signalling, was found to induce phosphorylation of Eps8, thus inhibiting the 


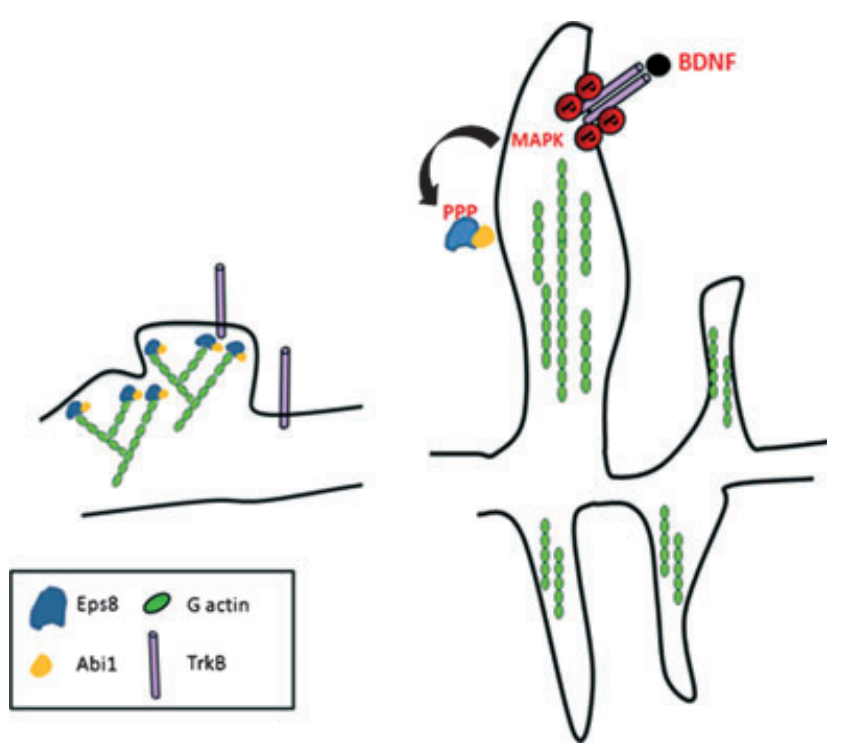

FIG. 3. Neurotrophic factors may control filopodia formation by regulating actin-capping proteins. A model depicting the effect of BDNF in regulating filopodia formation is shown. The neurotrophin, through MAPK activation, phosphorylates Eps8, inducing its detachment from actin barbed ends. This leads to actin elongation and filopodia formation. PPP, phosphorylation.

capping activity of the protein and causing its subcellular redistribution away from actin-rich structures (Menna et al., 2009) (Fig. 3A). Also, neuronal exposure to BDNF led to an alteration in actin dynamics, that is, a reduction in the half-time of recovery, as revealed by fluorescence recovery after photobleaching. The MAPK inhibitor PD98059 completely prevented this effect (Fig. 2D). Therefore, inhibition of actin-capping proteins and consequent alterations in actin dynamics appear to represent key events in filopodia formation. These data also open the possibility that, in response to an external signal such as BDNF, asymmetric deactivation of Eps8 by MAPK on one side of the cell or growth cone may lead to the formation of polarized filopodial protrusions, resulting in altered outgrowth and guidance (Lundquist, 2009). Interestingly, the NGF and BDNF pathways might work in concert, promoting, respectively, the matching between the formation of actin patches and the rate of emergence of filopodia from patches. On the other hand, lack of MAPK activation could decrease the possibility of filopodial extension from actin patches that are formed through the action of phosphoinositide 3-kinase (Ketschek et al., 2011).

\section{Loss of filopodia impairs neuritogenesis: involvement of actin-capping and anti-capping proteins}

In recent years, the concept has clearly emerged that the formation of axonal filopodia represents the first step in neuritogenesis. Neuritogenesis and collateral branching, that is, the processes by which postmitotic neurons extend long primary axons towards targets to form appropriate connections, are crucial processes for correct wiring of the brain and for the generation of appropriate synaptic networks (Garner et al., 2006; Korobova \& Svitkina, 2008). Ena/VASP family proteins bind actin and regulate the assembly of F-actin networks by antagonizing actin-capping proteins, by enhancing processive elongation, and by promoting the clustering and convergence of filaments at their barbed ends (Bear et al., 2002; Krause et al., 2003; Applewhite et al., 2007; Breitsprecher et al., 2008, 2011; Hansen \& Mullins, 2010). Notably, loss of the anti-capping proteins Ena and VASP causes a striking reduction in filopodia formation in stage 1 cortical neurons. Failure to form filopodia in Ena/VASP-deficient neurons induces a neurite initiation defect and blocks axon fibre tract formation in the cortex (Kwiatkowski et al., 2007). Therefore, reduction of filopodial extension, resulting from loss of Ena/VASP, may be the primary cause of the block in neuritogenesis. Interestingly, neuritogenesis in Ena/VASP-null neurons could be rescued by restoring filopodia formation through ectopic expression of the motor protein myosin $\mathrm{X}$ or the actin-nucleating protein $\mathrm{mDia} 2$, or by plating cells on the extracellular matrix protein laminin, which promotes the formation of filopodia-like actin-rich protrusions (Dent et al., 2007). These latter observations indicate that there are multiple and possibly independent molecular mechanisms to promote filopodial extension. In the same study, the authors also showed that neurite initiation requires microtubule extension into filopodia, suggesting that interactions between actin filament bundles and dynamic microtubules within filopodia are crucial for neuritogenesis (Dent et al., 2007). This is in line with the concept that actin bundles within filopodia serve as tracks for microtubule exploration (Schaefer et al., 2002) and that neurite formation occurs when actin filaments are destabilized, filopodia are extended, and microtubules invade filopodia (Georges et al., 2008). Therefore, a failure in filopodia formation may lead to a secondary defect in the microtubule-dependent functions required for neuritogenesis. At least one of the molecular components mediating the interaction between microtubules and filopodial F-actin has been identified as drebrin, an F-actin-associated protein that binds directly to the microtubule-binding protein EB3. Indeed, when this interaction is disrupted, the formation of growth cones and the extension of neurites are impaired (Geraldo et al., 2008). Whereas loss of anticapping proteins impairs neuritogenesis, the genetic absence of actincapping proteins enhances neuritogenesis. This has been shown in Eps8-deficient neurons, which, in culture, show a significantly higher number of neurites (Fig. 4). Thus, actin-capping and anti-capping proteins, through modulation of filopodia formation, appear to play a crucial role in neuritogenesis.

\section{From filopodia to synapses: actin-capping and anti-capping proteins at neuronal contacts}

Filopodia are thought to play an active role in synaptogenesis; in fact, they appear to guide the coordinated growth of presynaptic and postsynaptic partners (Dailey \& Smith, 1996; Ziv \& Smith, 1996; Fiala et al., 1998; Dunaevsky et al., 1999; Okabe et al., 2001; Evers et al., 2006). This concept is particularly well established in the case of dendritic filopodia, which participate in synaptic contact formation. These protrusions switch to more stable dendritic spines through the actions of synapse-inducing factors and neuronal activity (Jontes \& Smith, 2000; Bhatt et al., 2009; Hotulainen et al., 2009; Hotulainen and Hoogenraad, 2010). Although less well investigated, axonal filopodia, which emerge from the shaft of axonal branches and contain small synaptic vesicle clusters, are also thought to be involved in initiating synapse formation (Chang \& De Camilli, 2001). Accordingly, the actin-based motility of axonal filopodia is inversely correlated with contact with postsynaptic targets (Tashiro et al., 2003), whereas the emergence of axonal filopodia from varicosities enriched in synaptic vesicles favours assembly of the neuromuscular junction (Li et al., 2011).

Recent data have provided further evidence to support the longstanding concept that control of filopodia motility and number is only one aspect of establishing a synapse. Indeed, the extension of filopodial protrusions must be followed by the establishment of a 


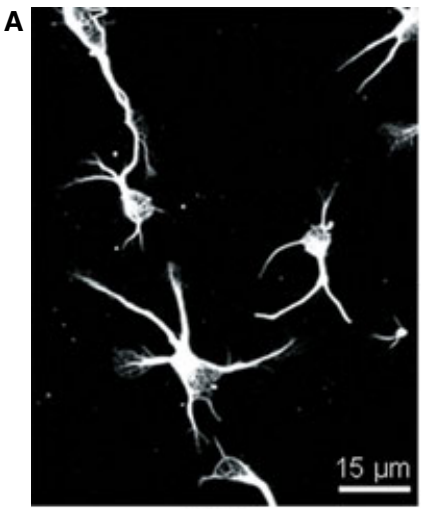

WT

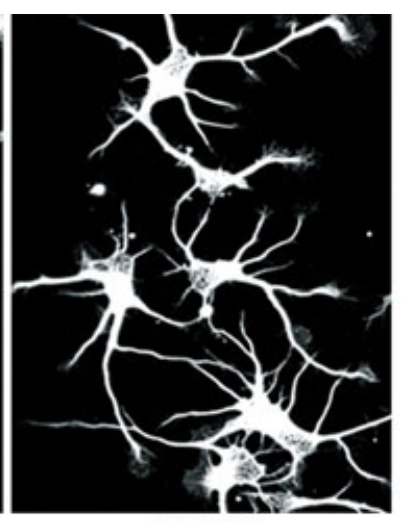

Eps8 KO
B

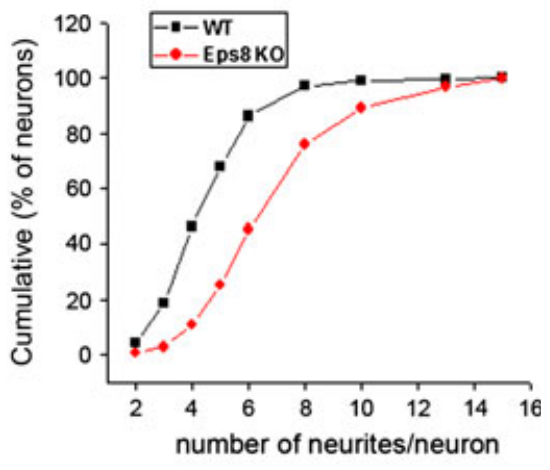

FIG. 4. Removal of the capping protein Eps8 enhances neuritogenesis. (A) $\beta$ III-tubulin staining of wild type (WT) and Eps8 knockout (KO) neurons reveals that protein removal induces a significant increase in neurite formation (reprinted from Menna et al., 2009). (B) Cumulative analysis of neurite number in WT and Eps 8 KO mice (Kolmogorov-Smirnov test, $P=0.001$ ).

transcellular interaction to stabilize the nascent contact. The two processes may be concomitantly controlled, as in the case of EphB, which, by increasing dendritic filopodia motility to initiate presynaptic and postsynaptic contact and by stabilizing nascent synapses through trans-synaptic interactions, mediates a filopodia-based synaptogenesis process (Ethell et al., 2001; Kayser et al., 2008). Arstikaitis et al. (2011), using a filopodia-inducing motif that is found in growthassociated protein-43, found enhanced filopodia numbers and motility, but a reduced probability of the formation of a stable axon-dendrite contact; conversely, expression of neuroligin-1 results in a decrease in filopodia motility, but an increase in the number of stable axonal contacts. Hence, enhancing filopodia formation and mobility may not necessarily lead to more stable axon-dendrite contacts. Rather, the production of stable synapses seems to be dependent on key members of the postsynaptic scaffolding complex (Arstikaitis et al., 2011).

It is presently not clear whether actin-capping and anti-capping proteins control the process of synaptogenesis from filopodia, modulating either their density or stability. Notably, however, actincapping and anti-capping proteins have been recently detected in dendritic spines. Platinum replica electron microscopy analysis has recently revealed that spine heads are characterized by a branched actin filament network containing the Arp2/3 complex and actincapping proteins, a cytoskeletal organization resembling the conventional lamellipodial structure (Hotulainen \& Hoogenraad, 2010). Notably, the actin-capping protein $\mathrm{CP}$, a regulator of actin filament growth, has been found recently to be essential for spine development and maturation, leading to functional synapses (Fan et al., 2011). Presynaptic boutons, as well as spine necks and bases, also contain a very similar branched network of actin cytoskeleton (Korobova \& Svitkina, 2010). Furthermore, it has been found that the anti-capping protein VASP is enriched in spine heads and synapses (Lin et al., 2010). VASP expression increases the size of the spine head and results in a significant increase in the amount of actin filaments and uncapped barbed ends available for further actin polymerization in spines (Lin et al., 2010). Ena/VASP was identified as a protein kinase A effector downstream of syndecan-2; in particular, syndecan-2 was found to activate, via neurofibromin, protein kinase A, which in turn phosphorylates Ena/VASP, thus promoting filopodium and spine formation (Lin et al., 2007). Interestingly, in spines, VASP elevates the amount of postsynaptic density scaffolding proteins, including PSD95, Homer, and Shank, and increases the number and retention of glutamate receptor type 1-containing AMPA receptors, thus ultimately

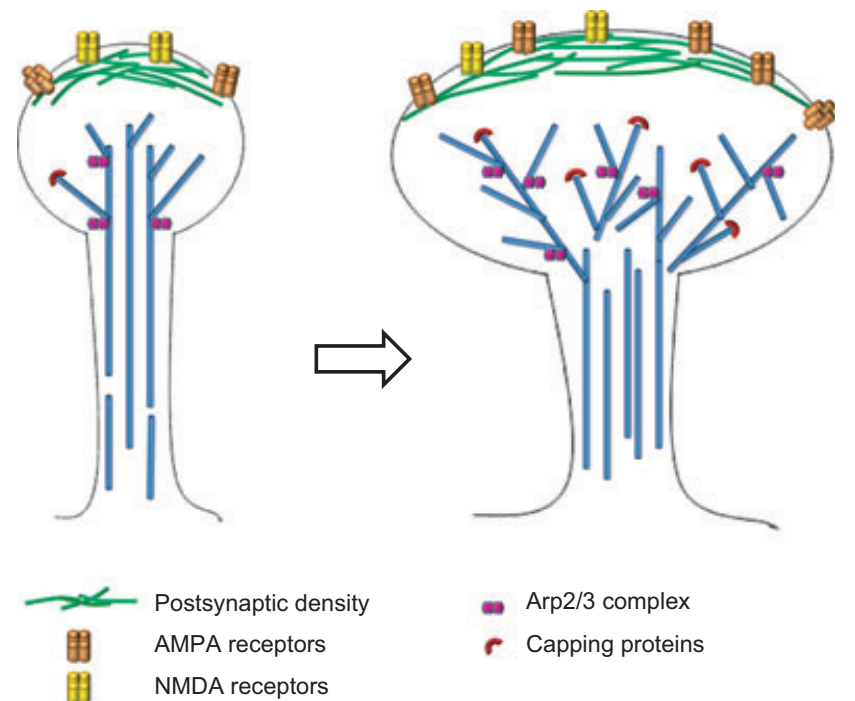

FIG. 5. Actin-capping and branching proteins in spine enlargement. The model depicts the possible involvement of actin-capping and branching proteins in favouring the spine head enlargement that occurs during long-term potentiation (based on Tada and Sheng, 2006; Hotulainen and Hoogenraad, 2010).

potentiating AMPA receptor-mediated synaptic transmission (Takahashi et al., 2003; Lin et al., 2010).

Notably, using fimbria-fornix transection as a model of memory deficit, coupled with a proteomic approach, Kitanishi and coworkers identified the F-actin-capping protein CapZ (Schafer \& Cooper, 1995) among the candidate proteins showing a reduction in level after fimbria-fornix transection. CapZ, which has been previously shown to regulate growth cone morphology and neurite outgrowth in cultured hippocampal neurons (Davis et al., 2009), was detected in dendritic spines of neurons in vitro, where its expression was reduced after silencing of neuronal activity by tetrodotoxin. Accordingly, unilateral administration of high-frequency stimuli to the medial perforant path in awake rats increased CapZ immunoreactivity (Kitanishi et al., 2010). Activity-dependent CapZ accumulation supported a possible role for the protein in the regulation of actin dynamics in response to synaptic inputs and eventually in synaptic plasticity. All together, these data suggest that regulation of capping activity is involved in 
synapse formation and postsynaptic stabilization, as well as in spine plasticity processes (Fig. 5).

\section{Conclusions and perspectives}

Actin-capping and anti-capping proteins are crucial regulators of actin dynamics. Recent studies have indicated that these proteins may be heavily involved in all stages of synaptogenesis, from the emergence of filopodia in developing neurons, through the processes of neuritogenesis and contact stabilization, to the structural changes occurring at the synapse during plasticity phenomena. In recent years, it has become evident that many psychiatric disorders, in particular autism and mental retardation, are accompanied by alterations in spine morphology and synapse number (Comery et al., 1997; Irwin et al., 2001; Bourgeron, 2009; Pfeiffer \& Huber, 2009; Hutsler \& Zhang, 2010; Pfeiffer et al., 2010). It is expected that future genetic studies will identify new mutations in actin-capping and anti-capping proteins that, by altering either the process of filopodia-driven synaptogenesis and/or spine actin regulation, may result in abnormalities of spine morphology and synapse dysfunction.

\section{Acknowledgements}

We thank R. Tomasoni and S. Zambetti (University of Milano) for discussion and for critically reading the manuscript. Work in our laboratories is supported by grants from the European Union Seventh Framework Programme under grant agreement no. HEALTH-F2-2009-241498 (EUROSPIN project), from Compagnia di San Paolo (2008-2207) and from the Italian Ministry of Education, Universities and Research (MIUR-PRIN) (2008 2008T4ZCNL) to M. Matteoli, and from the Associazione Italiana per la Ricerca sul Cancro (AIRC), the International Association for Cancer Research (AICR), CARIPLO Foundations, the Italian Ministry of Education, Universities and Research (MIUR-PRIN), the Italian Ministry of Health and the European Research Council to G. Scita.

\section{Abbreviations}

BNDF, brain-derived neurotrophic factor; CP, capping protein; IRSp53, insulin receptor tyrosine kinases substrate of $53 \mathrm{kDa}$; MAPK, mitogen-activated protein kinase; NGF, nerve growth factor; VASP, vasodilator-stimulated phosphoprotein.

\section{References}

Abbott, M.A., Wells, D.G. \& Fallon, J.R. (1999) The insulin receptor tyrosine kinase substrate p58/53 and the insulin receptor are components of CNS synapses. J. Neurosci., 19, 7300-7308.

Akin, O. \& Mullins, R.D. (2008) Capping protein increases the rate of actinbased motility by promoting filament nucleation by the Arp $2 / 3$ complex Cell, 133, 841-851.

Applewhite, D.A., Barzik, M., Kojima, S., Svitkina, T.M., Gertler, F.B. \& Borisy, G.G. (2007) Ena/VASP proteins have an anti-capping independent function in filopodia formation. Mol. Biol. Cell, 18, 2579-2591.

Arstikaitis, P., Gauthier-Campbell, C., Huang, K., El-Husseini, A. \& Murphy, T.H. (2011) Proteins that promote filopodia stability, but not number, lead to more axonal-dendritic contacts. PLOS ONE, 6, e16998.

Bear, J.E., Svitkina, T.M., Krause, M., Schafer, D.A., Loureiro, J.J., Strasser, G.A., Maly, I.V., Chaga, O.Y., Cooper, J.A., Borisy, G.G. \& Gertler, F.B. (2002) Antagonism between Ena/VASP proteins and actin filament capping regulates fibroblast motility. Cell, 109, 509-521.

Bhatt, D.H., Zhang, S. \& Gan, W.B. (2009) Dendritic spine dynamics. Аnnи. Rev. Physiol., 71, 261-282.

Block, J., Stradal, T.E., Hanisch, J., Geffers, R., Kostler, S.A., Urban, E., Small, J.V., Rottner, K. \& Faix, J. (2008) Filopodia formation induced by active mDia2/Drf3. J. Microsc., 231, 506-517.

Bourgeron, T. (2009) A synaptic trek to autism. Curr. Opin. Neurobiol., 19, 231-234.

Breitsprecher, D., Kiesewetter, A.K., Linkner, J., Urbanke, C., Resch, G.P., Small, J.V. \& Faix, J. (2008) Clustering of VASP actively drives processive, WH2 domain-mediated actin filament elongation. EMBO J., 27, 2943-2954.

Breitsprecher, D., Kiesewetter, A.K., Linkner, J., Vinzenz, M., Stradal, T.E., Small, J.V., Curth, U., Dickinson, R.B. \& Faix, J. (2011) Molecular mechanism of Ena/VASP-mediated actin-filament elongation. EMBO J., 30, 456-467.

Bruel-Jungerman, E., Davis, S. \& Laroche, S. (2007) Brain plasticity mechanisms and memory: a party of four. Neuroscientist, 13, 492-505.

Chang, S. \& De Camilli, P. (2001) Glutamate regulates actin-based motility in axonal filopodia. Nat. Neurosci., 4, 787-793.

Chen, T.J., Gehler, S., Shaw, A.E., Bamburg, J.R. \& Letourneau, P.C. (2006) $\mathrm{Cdc} 42$ participates in the regulation of $\mathrm{ADF} /$ cofilin and retinal growth cone filopodia by brain derived neurotrophic factor. J. Neurobiol., 66, 103-114.

Chesarone, M.A. \& Goode, B.L. (2009) Actin nucleation and elongation factors: mechanisms and interplay. Curr. Opin. Cell Biol., 21, 28-37.

Chklovskii, D.B., Mel, B.W. \& Svoboda, K. (2004) Cortical rewiring and information storage. Nature, 431, 782-788.

Comery, T.A., Harris, J.B., Willems, P.J., Oostra, B.A., Irwin, S.A., Weiler, I.J. \& Greenough, W.T. (1997) Abnormal dendritic spines in fragile X knockout mice: maturation and pruning deficits. Proc. Natl. Acad. Sci. USA, 94, 54015404

Craig, A.M., Graf, E.R. \& Linhoff, M.W. (2006) How to build a central synapse: clues from cell culture. Trends Neurosci., 29, 8-20.

Croce, A., Cassata, G., Disanza, A., Gagliani, M.C., Tacchetti, C., Malabarba, M.G., Carlier, M.F., Scita, G., Baumeister, R. \& Di Fiore, P.P. (2004) A novel actin barbed-end-capping activity in EPS-8 regulates apical morphogenesis in intestinal cells of Caenorhabditis elegans. Nat. Cell Biol., 6, 1173-1179.

Dailey, M.E. \& Smith, S.J. (1996) The dynamics of dendritic structure in developing hippocampal slices. J. Neurosci., 16, 2983-2994.

Davis, D.A., Wilson, M.H., Giraud, J., Xie, Z., Tseng, H.C., England, C., Herscovitz, H., Tsai, L.H. \& Delalle, I. (2009) Capzb2 interacts with betatubulin to regulate growth cone morphology and neurite outgrowth. PLoS Biol., 7, e1000208.

Dent, E.W., Kwiatkowski, A.V., Mebane, L.M., Philippar, U., Barzik, M., Rubinson, D.A., Gupton, S., Van Veen, J.E., Furman, C., Zhang, J., Alberts, A.S., Mori, S. \& Gertler, F.B. (2007) Filopodia are required for cortical neurite initiation. Nat. Cell Biol., 9, 1347-1359.

Disanza, A., Carlier, M.F., Stradal, T.E., Didry, D., Frittoli, E., Confalonieri, S. Croce, A., Wehland, J., Di Fiore, P.P. \& Scita, G. (2004) Eps8 controls actinbased motility by capping the barbed ends of actin filaments. Nat. Cell Biol., 6. $1180-1188$

Disanza, A., Mantoani, S., Hertzog, M., Gerboth, S., Frittoli, E., Steffen, A., Berhoerster, K., Kreienkamp, H.J., Milanesi, F., Di Fiore, P.P., Ciliberto, A., Stradal, T.E. \& Scita, G. (2006) Regulation of cell shape by Cdc42 is mediated by the synergic actin-bundling activity of the Eps8-IRSp53 complex. Nat. Cell Biol., 8, 1337-1347.

Drees, F. \& Gertler, F.B. (2008) Ena/VASP: proteins at the tip of the nervous system. Curr. Opin. Neurobiol., 18, 53-59.

Dunaevsky, A., Tashiro, A., Majewska, A., Mason, C. \& Yuste, R. (1999) Developmental regulation of spine motility in the mammalian central nervous system. Proc. Natl. Acad. Sci. USA, 96, 13438-13443.

Ethell, I.M., Irie, F., Kalo, M.S., Couchman, J.R., Pasquale, E.B. \& Yamaguchi, Y. (2001) EphB/syndecan-2 signaling in dendritic spine morphogenesis. Neuron, 31, 1001-1013.

Evers, J.F., Muench, D. \& Duch, C. (2006) Developmental relocation of presynaptic terminals along distinct types of dendritic filopodia. Dev. Biol., 297, 214-227.

Faix, J., Breitsprecher, D., Stradal, T.E. \& Rottner, K. (2009) Filopodia: complex models for simple rods. Int. J. Biochem. Cell Biol., 41, 1656-1664.

Fan, Y. \& Tang, X., Vitriol, E., Chen, G. \& Zheng, J.Q. (2011) Actin capping protein is required for dendritic spine development and synapse formation. J. Neurosci., 31, 10228-10233.

Fass, J., Gehler, S., Sarmiere, P., Letourneau, P. \& Bamburg, J.R. (2004) Regulating filopodial dynamics through actin-depolymerizing factor/cofilin. Anat. Sci. Int., 79, 173-183.

Fiala, J. C., Feinberg, M., Popov, V. \& Harris, K.M. (1998) Synaptogenesis via dendritic filopodia in developing hippocampal area CA1. J. Neurosci., 18, 8900-8911.

Fu, M. \& Zuo, Y. (2011) Experience-dependent structural plasticity in the cortex. Trends Neurosci., 34, 177-187.

Garner, C.C., Waites, C.L. \& Ziv, N.E. (2006) Synapse development: still looking for the forest, still lost in the trees. Cell Tissue Res., 326, 249-262.

Gehler, S., Shaw, A.E., Sarmiere, P.D., Bamburg, J.R. \& Letourneau, P.C. (2004) Brain-derived neurotrophic factor regulation of retinal growth cone 
filopodial dynamics is mediated through actin depolymerizing factor/cofilin. J. Neurosci., 24, 10741-10749.

Georges, P.C., Hadzimichalis, N.M., Sweet, E.S. \& Firestein, B.L. (2008) The yin-yang of dendrite morphology: unity of actin and microtubules. Mol. Neurobiol., 38, 270-284.

Geraldo, S. \& Gordon-Weeks, P.R. (2009) Cytoskeletal dynamics in growthcone steering. J. Cell Sci., 122, 3595-3604.

Geraldo, S., Khanzada, U.K., Parsons, M., Chilton, J.K. \& Gordon-Weeks, P.R. (2008) Targeting of the F-actin-binding protein drebrin by the microtubule plus-tip protein EB3 is required for neuritogenesis. Nat. Cell Biol., 10, 11811189.

Gupton, S.L., Eisenmann, K., Alberts, A.S. \& Waterman-Storer, C.M. (2007) $\mathrm{mDia} 2$ regulates actin and focal adhesion dynamics and organization in the lamella for efficient epithelial cell migration. J. Cell Sci., 120, 3475-3487.

Hansen, S.D. \& Mullins, R.D. (2010) VASP is a processive actin polymerase that requires monomeric actin for barbed end association. J. Cell Biol., 191, $571-584$

Heiman, M.G. \& Shaham, S. (2010) Twigs into branches: how a filopodium becomes a dendrite. Curr. Opin. Neurobiol., 20, 86-91.

Hotulainen, P. \& Hoogenraad, C.C. (2010). Actin in dendritic spines: connecting dynamics to function. J. Cell Biol., 189, 619-629.

Hotulainen, P., Llano, O., Smirnov, S., Tanhuanpaa, K., Faix, J., Rivera, C. \& Lappalainen, P. (2009) Defining mechanisms of actin polymerization and depolymerization during dendritic spine morphogenesis. J. Cell Biol., 185, 323-339.

Hutsler, J.J. \& Zhang, H. (2010) Increased dendritic spine densities on cortical projection neurons in autism spectrum disorders. Brain Res., 1309, 83-94.

Irwin, S.A., Patel, B., Idupulapati, M., Harris, J.B., Crisostomo, R.A., Larsen, B.P., Kooy, F., Willems, P.J., Cras, P., Kozlowski, P.B., Swain, R.A., Weiler, I.J. \& Greenough, W.T. (2001) Abnormal dendritic spine characteristics in the temporal and visual cortices of patients with fragile-X syndrome: a quantitative examination. Am. J. Med. Genet., 98, 161-167.

Jontes, J.D. \& Smith, S.J. (2000) Filopodia, spines, and the generation of synaptic diversity. Neuron, 27, 11-14.

Jourdain, P., Fukunaga, K. \& Muller, D. (2003) Calcium/calmodulindependent protein kinase II contributes to activity-dependent filopodia growth and spine formation. J. Neurosci., 23, 10645-10649.

Kayser, M.S., Nolt, M.J. \& Dalva, M.B. (2008) EphB receptors couple dendritic filopodia motility to synapse formation. Neuron, 59, 56-69.

Ketschek, A. \& Gallo, G. (2010) Nerve growth factor induces axonal filopodia through localized microdomains of phosphoinositide 3-kinase activity that drive the formation of cytoskeletal precursors to filopodia. J. Neurosci., $\mathbf{3 0}$, 12185-12197.

Ketschek, A., Spillane, M. \& Gallo, G. (2011) Mechanism of NGF-induced formation of axonal filopodia: NGF turns up the volume, but the song remains the same? Commun. Integr. Biol., 4, 55-58.

Kitanishi, T., Sakai, J., Kojima, S., Saitoh, Y., Inokuchi, K., Fukaya, M., Watanabe, M., Matsuki, N. \& Yamada, M.K. (2010) Activity-dependent localization in spines of the F-actin capping protein CapZ screened in a rat model of dementia. Genes Cells, 15, 737-747.

Knott, G. \& Holtmaat, A. (2008) Dendritic spine plasticity - current understanding from in vivo studies. Brain Res. Rev., 58, 282-289.

Koleske, A.J. (2003) Do filopodia enable the growth cone to find its way? Sci. STKE, 183, pe20.

Korobova, F. \& Svitkina, T. (2008) Arp2/3 complex is important for filopodia formation, growth cone motility, and neuritogenesis in neuronal cells. Mol. Biol. Cell, 19, 1561-1574.

Korobova, F. \& Svitkina, T. (2010) Molecular architecture of synaptic actin cytoskeleton in hippocampal neurons reveals a mechanism of dendritic spine morphogenesis. Mol. Biol. Cell, 21, 165-176.

Krause, M., Dent, E.W., Bear, J.E., Loureiro, J.J. \& Gertler, F.B. (2003) Ena/VASP proteins: regulators of the actin cytoskeleton and cell migration. Annu. Rev. Cell Dev. Biol., 19, 541-564.

Kwiatkowski, A.V., Rubinson, D.A., Dent, E.W., Edward van Veen, J., Leslie, J.D., Zhang, J., Mebane, L.M., Philippar, U., Pinheiro, E.M., Burds, A.A., Bronson, R.T., Mori, S., Fassler, R. \& Gertler, F.B. (2007) Ena/VASP is required for neuritogenesis in the developing cortex. Neuron, 56, 441-455.

Le Clainche, C. \& Carlier, M.F. (2008) Regulation of actin assembly associated with protrusion and adhesion in cell migration. Physiol. Rev., 88, 489-513.

Li, P.P., Chen, C., Lee, C.W., Madhavan, R. \& Peng, H.B. (2011) Axonal filopodial asymmetry induced by synaptic target. Mol. Biol. Cell, 22, 24802490 .
Lin, Y.L., Nehban, C.A., Hong, C.J. \& Hsueh, Y.P. (2007) Syndecan-2 induces filopodia and dendritic spine formation via the neurofibromin-PKAEna/VASP pathway. J. Cell Biol., 177, 829-841.

Lin, Y.L., Lei, Y.T., Anderson, B.R. \& Webb, D.J. (2011) Vasodilatorstimulated phosphoprotein (VASP) induces actin assembly in dendritic spines to promote their development and potentiate synaptic strength. J. Biol. Chem., 285, 36010-36020.

Lu, M., Witke, W., Kwiatkowski, D.J. \& Kosik, K.S. (1997) Delayed retraction of filopodia in gelsolin null mice. J. Cell Biol., 138, 1279-1287.

Lundquist, E.A. (2009) The finer points of filopodia. PLoS Biol., 7, e1000142.

Luscher, C., Nicoll, R.A., Malenka, R.C. \& Muller, D. (2000) Synaptic plasticity and dynamic modulation of the postsynaptic membrane. Nat. Neurosci., 3, 545-550.

Mai, J., Fok, L., Gao, H., Zhang, X. \& Poo, M.M. (2009) Axon initiation and growth cone turning on bound protein gradients. J. Neurosci., 29, 74507458.

Matteoli, M., Coco, S., Schenk, U. \& Verderio, C. (2004) Vesicle turnover in developing neurons: how to build a presynaptic terminal. Trends Cell Biol., 14, 133-140.

Mattila, P.K. \& Lappalainen, P. (2008) Filopodia: molecular architecture and cellular functions. Nat. Rev. Mol. Cell Biol., 9, 446-454.

McAllister, A.K. (2007) Dynamic aspects of CNS synapse formation. Annu. Rev. Neurosci., 30, 425-450.

Mejillano, M.R., Kojima, S., Applewhite, D.A., Gertler, F.B., Svitkina, T.M. \& Borisy, G.G. (2004) Lamellipodial versus filopodial mode of the actin nanomachinery: pivotal role of the filament barbed end. Cell, 118, 363373.

Mellor, H. (2010) The role of formins in filopodia formation. Biochim. Biophys. Acta, 1803, 191-200.

Menna, E., Disanza, A., Cagnoli, C., Schenk, U., Gelsomino, G., Frittoli, E., Hertzog, M., Offenhauser, N., Sawallisch, C., Kreienkamp, H.J., Gertler, F.B., Di Fiore, P.P., Scita, G. \& Matteoli, M. (2009) Eps8 regulates axonal filopodia in hippocampal neurons in response to brain-derived neurotrophic factor (BDNF). PLoS Biol., 7, e1000138.

Mogilner, A. \& Rubinstein, B. (2005) The physics of filopodial protrusion. Biophys. J., 89, 782-795.

Nikonenko, I., Jourdain, P. \& Muller, D. (2003) Presynaptic remodeling contributes to activity-dependent synaptogenesis. J. Neurosci., 23, 84988505.

Oda, K., Shiratsuchi, T., Nishimori, H., Inazawa, J., Yoshikawa, H., Taketani, Y., Nakamura, Y. \& Tokino, T. (1999) Identification of BAIAP2 (BAIassociated protein 2), a novel human homologue of hamster IRSp53, whose SH3 domain interacts with the cytoplasmic domain of BAI1. Cytogenet. Cell Genet., 84, 75-82.

Offenhauser, N., Borgonovo, A., Disanza, A., Romano, P., Ponzanelli, I., Iannolo, G., Di Fiore, P.P. \& Scita, G. (2004) The eps8 family of proteins links growth factor stimulation to actin reorganization generating functional redundancy in the Ras/Rac pathway. Mol. Biol. Cell, 15, 91-98.

Okabe, S., Miwa, A. \& Okado, H. (2001) Spine formation and correlated assembly of presynaptic and postsynaptic molecules. J. Neurosci., 21, 61056114.

Ono, S. (2007) Mechanism of depolymerization and severing of actin filaments and its significance in cytoskeletal dynamics. Int. Rev. Cytol., 258, 1-82.

Pellegrin, S. \& Mellor, H. (2005) The Rho family GTPase Rif induces filopodia through mDia2. Curr. Biol., 15, 129-133.

Pfeiffer, B.E. \& Huber, K.M. (2009) The state of synapses in fragile X syndrome. Neuroscientist, 15, 549-567.

Pfeiffer, B.E., Zang, T., Wilkerson, J.R., Taniguchi, M., Maksimova, M.A. Smith, L.N., Cowan, C.W. \& Huber, K.M. (2010) Fragile X mental retardation protein is required for synapse elimination by the activitydependent transcription factor MEF2. Neuron, 66, 191-197.

Portera-Cailliau, C., Pan, D.T. \& Yuste, R. (2003) Activity-regulated dynamic behavior of early dendritic protrusions: evidence for different types of dendritic filopodia. J. Neurosci., 23, 7129-7142.

Ridley, A.J. (2011) Life at the leading edge. Cell, 145, 1012-1022.

Schaefer, A.W., Kabir, N. \& Forscher, P. (2002) Filopodia and actin arcs guide the assembly and transport of two populations of microtubules with unique dynamic parameters in neuronal growth cones. J. Cell Biol., 158, $139-152$.

Schafer, D.A. \& Cooper, J.A. (1995) Control of actin assembly at filament ends. Annu. Rev. Cell Dev. Biol., 11, 497-518.

Schirenbeck, A., Bretschneider, T., Arasada, R., Schleicher, M. \& Faix, J. (2005) The Diaphanous-related formin dDia2 is required for the formation and maintenance of filopodia. Nat. Cell Biol., 7, 619-625. 
Scita, G., Confalonieri, S., Lappalainen, P. \& Suetsugu, S. (2008) IRSp53: crossing the road of membrane and actin dynamics in the formation of membrane protrusions. Trends Cell Biol., 18, 52-60.

Svitkina, T.M., Bulanova, E.A., Chaga, O.Y., Vignjevic, D.M., Kojima, S., Vasiliev, J.M. \& Borisy, G.G. (2003) Mechanism of filopodia initiation by reorganization of a dendritic network. J. Cell Biol., 160, 409-421.

Tada, T. \& Sheng, M. (2006) Molecular mechanisms of dendritic spine morphogenesis. Curr. Opin. Neurobiol., 16, 95-101.

Takahashi, T., Svoboda, K. \& Malinow, R. (2003) Experience strengthening transmission by driving AMPA receptors into synapses. Science, 299, 1585 1588

Tashiro, A., Dunaevsky, A., Blazeski, R., Mason, C.A. \& Yuste, R. (2003) Bidirectional regulation of hippocampal mossy fiber filopodial motility by kainate receptors: a two-step model of synaptogenesis. Neuron, 38, 773784.

Vaggi, F. \& Disanza, A., Milanesi, F., Di Fiore, P.P., Menna, E., Matteoli, M., Gov, N.S., Scita, G. \& Ciliberto, A. (2011) The Eps8/IRSp53/VASP network differentially controls actin capping and bundling in filopodia formation PLoS Comput Biol., 7, e1002088, doi:10.1371/journal.pcbi.1002088.

Valiente, M. \& Martini, F.J. (2009) Migration of cortical interneurons relies on branched leading process dynamics. Cell Adh. Migr., 3, 278-280.

Vignjevic, D., Peloquin, J. \& Borisy, G.G. (2006) In vitro assembly of filopodia-like bundles. Methods Enzymol., 406, 727-739.

Wong, W.T., Faulkner-Jones, B.E., Sanes, J.R. \& Wong, R.O. (2000) Rapid dendritic remodeling in the developing retina: dependence on neurotransmission and reciprocal regulation by Rac and Rho. J. Neurosci., 20, 50245036.

Yang, C., Czech, L., Gerboth, S., Kojima, S., Scita, G. \& Svitkina, T. (2007) Novel roles of formin $\mathrm{mDia} 2$ in lamellipodia and filopodia formation in motile cells. PLoS Biol., 5, e317.

Yuste, R. \& Bonhoeffer, T. (2004) Genesis of dendritic spines: insights from ultrastructural and imaging studies. Nat. Rev. Neurosci., 5, 24-34.

Ziv, N.E. \& Smith, S.J. (1996) Evidence for a role of dendritic filopodia in synaptogenesis and spine formation. Neuron, 17, 91-102. 